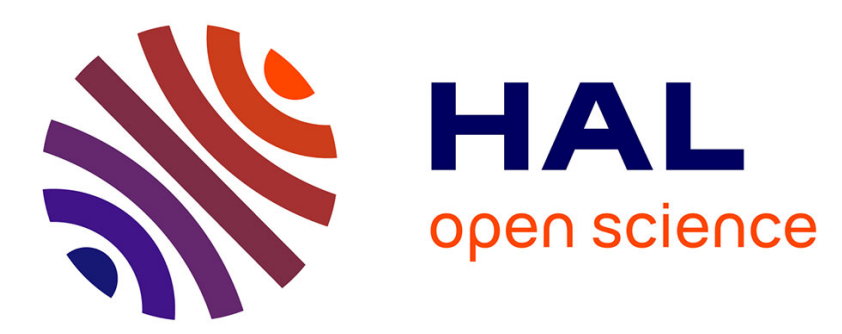

\title{
Design of Bragg gratings having negative group time delay for continuum generation
}

\author{
Adrian Grigore Iordachescu, Joël Jacquet
}

\section{To cite this version:}

Adrian Grigore Iordachescu, Joël Jacquet. Design of Bragg gratings having negative group time delay for continuum generation. SPIE Photonics West, Jan 2012, San Francisco, United States. 10.1117/12.909194. hal-00684727

\section{HAL Id: hal-00684727}

https://hal-centralesupelec.archives-ouvertes.fr/hal-00684727

Submitted on 19 Mar 2020

HAL is a multi-disciplinary open access archive for the deposit and dissemination of scientific research documents, whether they are published or not. The documents may come from teaching and research institutions in France or abroad, or from public or private research centers.
L'archive ouverte pluridisciplinaire HAL, est destinée au dépôt et à la diffusion de documents scientifiques de niveau recherche, publiés ou non, émanant des établissements d'enseignement et de recherche français ou étrangers, des laboratoires publics ou privés.

\section{(c)(1)}

Distributed under a Creative Commons Attribution| 4.0 International License 


\title{
Design of Bragg gratings having negative group time delay for continuum generation
}

\author{
G.A. Iordachescu, J. Jacquet \\ OPTEL, SUPELEC, 2 rue Edouard Branly, 57070 Metz, France
}

\begin{abstract}
In this paper we present the simulation results of two different methods of grating engineering (Genetic Algorithm and Discrete Layer Peeling) used for the synthesis of Bragg Gratings with a negative Group Time Delay. The physical structures of the resulting gratings, as well as their reflection spectra, are displayed for different initial parameters. These gratings could be employed (as it will be demonstrated) as cavity reflectors for the generation of a continuum laser emission by other means than those of nonlinear optics.
\end{abstract}

Keywords: chirped Bragg gratings, Genetic Algorithm, Discrete Layer Peeling, negative Group Time Delay, Continuum emission

\section{INTRODUCTION}

Starting from the concept of Continuum cavity developed by Bergonzo et $\mathrm{al}^{1}$ for the case of wide tuning range lasers, this paper will try to extend the same principle for the case of the widening of resonant modes of a Fabry-Perot cavity. The basic structure is shown in Fig. 1 and it consists of a simple Fabry-Perot cavity in which one of the mirrors was replaced by a Bragg grating supposed to expose a high reflectivity and a negative Group Time Delay (GTD) in the desired bandwidth. The purpose of this article is to describe the methods and steps used in trying to create such gratings. To synthesize such a Bragg grating two methods already presented in the literature are tried: Genetic Algorithm ${ }^{2}$ and Discrete Layer Peeling method ${ }^{3}$. The later of these methods could not be applied directly, as it suits only the design of the gratings for which just the amplitude condition is important. The grating design addressed in this paper needs a phase condition as well, so a further discussion on Feced ${ }^{4}$ and zero-padding ${ }^{5}$ methods is needed. The reflection spectra of all structures presented in this paper were realized by the Transfer Matrix Method ${ }^{6,7}$.

\section{THEORETICAL MODEL}

A filter formed by a simple Fabry-Perot (F-P) cavity was used, in which one of the mirrors was taken to be a Bragg grating, as shown in Fig. 1, which has the purpose of widening the resonant modes which would normally occur in the bandwidth of resonance. In the most general case, the refractive index of the grating is supposed to vary in a non regular manner. In this figure, the total round-trip phase shift has been separated in two terms, one term corresponding to the path through the cavity $\left(\varphi_{\mathrm{F}-\mathrm{P}}\right)$ and the other to the medium path length of radiation in the Bragg grating $\left(\varphi_{\mathrm{BG}}\right)$. An ideal grating would not just enlarge the modes in the cavity, but would be able to cancel them completely, making the structure exhibit a similar functionality to that of a continuum laser. In Fig.1 M1 is a simple dielectric mirror, introducing a phase difference of $\pm \pi$ during reflection which, being constant with the radiation wavelength, can be neglected in further calculus. In the same figure, the round-trip phase shift has been separated in two terms, one term corresponding to the path through the cavity $\left(\varphi_{\mathrm{F}-\mathrm{P}}\right)$ and the other to the medium path length of radiation in the Bragg grating $\left(\varphi_{\mathrm{BG}}\right)$. In a usual F-P cavity the sum of the two is a multiple of $2 \pi$ for every wavelength $\lambda$ corresponding to a maximum of transmission. In our case, $\varphi_{\mathrm{BG}}$ must have such a value that the total round trip phase shift $\left(\varphi_{\mathrm{F}-\mathrm{P}}+\varphi_{\mathrm{BG}}\right)$ should remain constant on a large enough bandwidth. In other words (1) the variation of $\varphi_{\mathrm{BG}}$ must cancel the variation of $\varphi_{\mathrm{F}-\mathrm{P}}$ with $\lambda$.

$$
\mathrm{d} \varphi_{\mathrm{BG}} / \mathrm{d} \lambda=-\mathrm{d} \varphi_{\mathrm{F}-\mathrm{P}} / \mathrm{d} \lambda
$$

If (1) is true for a large enough bandwidth, our structure will show a Continuum behavior. Because the Group Time Delay (GTD) of radiation through a structure is proportional to $\mathrm{d} \varphi / \mathrm{d} \lambda$, where $\varphi$ is the phase shift during reflection or transmission, then it can be stated from (1) that the GTD of the designed grating must have the same absolute value and opposite sign comparing to the GTD of radiation through the cavity. This condition must be true no matter the phase 
formalism adopted. In the proceeding calculus and Transfer Matrix Method ${ }^{6,7}$ (TMM) simulations, the phase shift of radiation after propagation on a distance $L$ will be considered equal to $\varphi(L)=-2 \pi n L / \lambda$. In this phase formalism, the GTD of the cavity will always be positive. This implies from (1) that the GTD of the Bragg grating (obtained by TMM) should be correspondingly negative in the desired bandwidth:

$$
\mathrm{d} \varphi_{\mathrm{BG}} / \mathrm{d} \lambda<0
$$

A Bragg grating which respects condition (1) will be called from now on a cavity-matched grating, as the group time delay after reflection is designed to oppose the GTD of the cavity on a large enough bandwidth (large enough so the effects could be measured). The effect of using such a grating as a reflector in the cavity could be observed in Fig. 2, by comparing the transmission spectra of the left figure (where an uniform grating has been used) with the right one (in which a cavity-matched grating was used). The first reflection on the left mirror (when light is entering the cavity) was neglected for normalization purposes. It is clearly seen that even though the length of the cavity remains the same, the effect of introducing a negative GTD grating (with an absolute value matching the cavity's GTD) is as if the cavity became much shorter (obtaining thus a widening of the F-P modes).

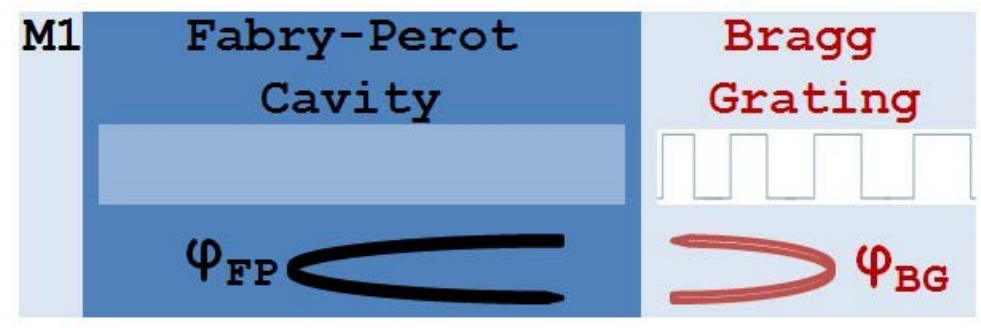

Figure 1. Schematic of a Continuum resonant cavity, in which the total round trip phase has been divided between the Bragg grating $\left(\varphi_{\mathrm{BG}}\right)$ and the rest of the cavity $\left(\varphi_{\mathrm{F}-\mathrm{P}}\right)$
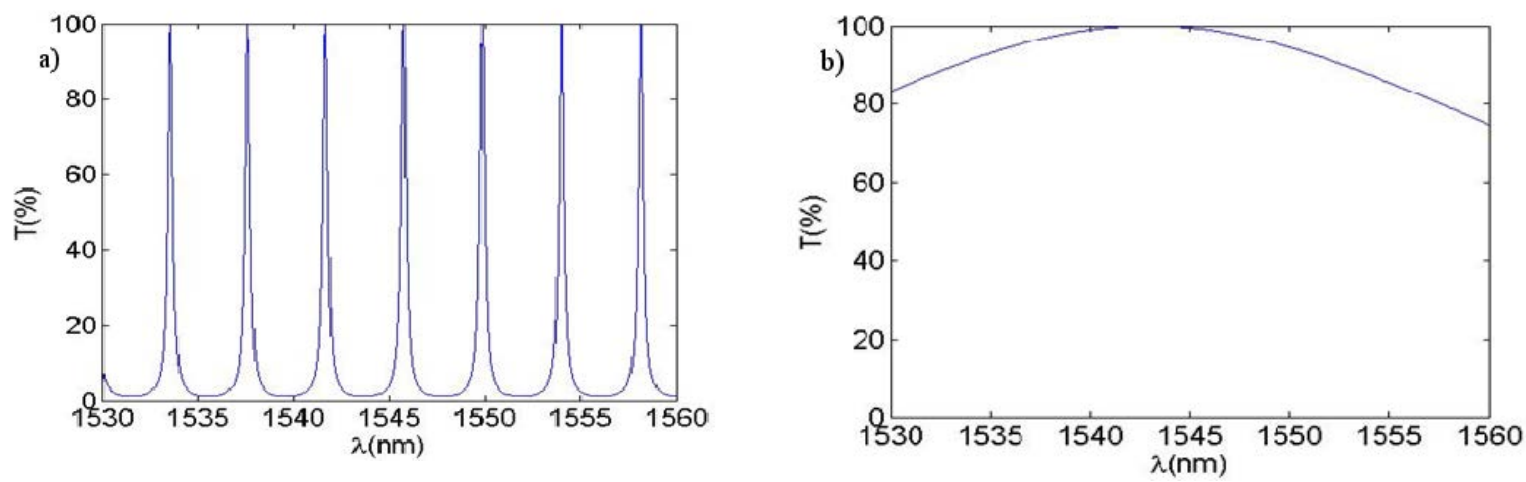

Figure 2. Simulation of transmission spectra of F-P cavity of figure 1, when the Bragg grating used as a mirror is: a) uniform; b) with a negative GTD, almost perfectly matched with the GTD of the cavity. For both of the pictures, the first reflection (on mirror M1) is neglected for normalization.

The two algorithms described next are employed for the design of Bragg gratings with high reflectivities and for which the condition (2) is true. It should be taken intro consideration here that the conditions (1) and (2) are not equivalent. Indeed, having a negative GTD for the grating - as implied by the condition (2) - does not guarantee a perfect canceling of the GTD of the cavity - as implied by (1). Because the cavity's GTD is positive and approximately constant (if the bandwidth is under $30 \mathrm{~nm}$ for a central wavelength of $1545 \mathrm{~nm}$ ) than the negative GTD of the grating should also keep constant in the desired bandwidth for the condition (1) to be fully respected. Nevertheless, in the following synthesis methods of the Bragg gratings, only condition (2) will be imposed. The necessity for using synthesis algorithms comes from the fact that no conventional gratings (uniform Bragg gratings, Linearly Chirped Gratings, etc) exhibit a negative GTD in the reflection bandwidth. For example, in the case of Linearly Chirped Gratings (similar to the one used by Bergonzo et al. ${ }^{1}$ ) three parameters (chirp, grating length and coupling coefficient) were independently varied without success. As an example, Fig. 3 shows the normalized GTD value as a function of chirp for a common Linearly Chirped 
Grating (LCG) of length $15 \mathrm{~cm}$ and coupling coefficient $k=80 \mathrm{~cm}^{-1}$. It can be noticed that no matter the chirp used, the GTD will always be positive. Correspondingly, in Fig. 4 a simulation of the GTD as a function of the coupling coefficient was realized for a LCG of length $15 \mathrm{~cm}$ and chirp C=100 $\mathrm{nm} / \mathrm{cm}$. It can be again noticed that the GTD remains positive, even if an asymptotical approach to zero was realized. In fact, there is no uniform Bragg grating or linearly chirped gratings which can be used as an effective reflector for the structure in Fig. 1, thus the necessity of designing the gratings by the algorithms described next.

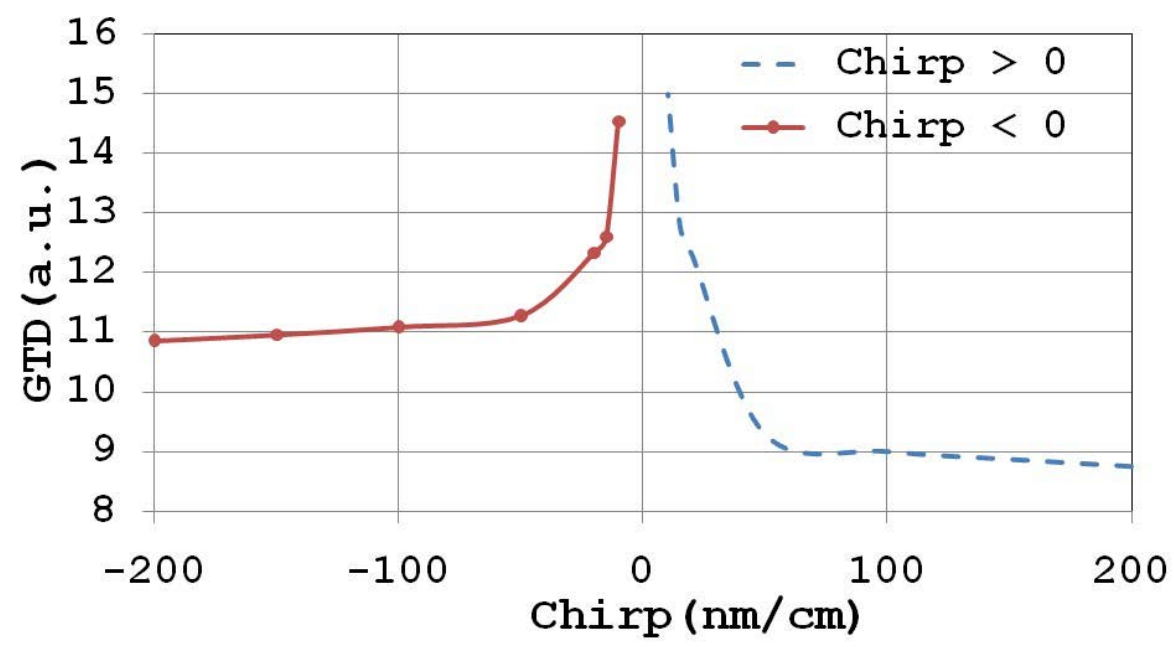

Figure 3. GTD as a function of chirp for a LCG of length $15 \mathrm{~cm}$ and coupling coefficient $k=80 \mathrm{~cm}^{-1}$. The values of GTD are normalized so that each unit corresponds to a phase derivative $d \varphi_{\mathrm{BG}} / \mathrm{d} \lambda$ of $1 \mathrm{rad} / \mathrm{nm}$. The negative chirp is presented in solid line; the positive chirp is in dashed line.

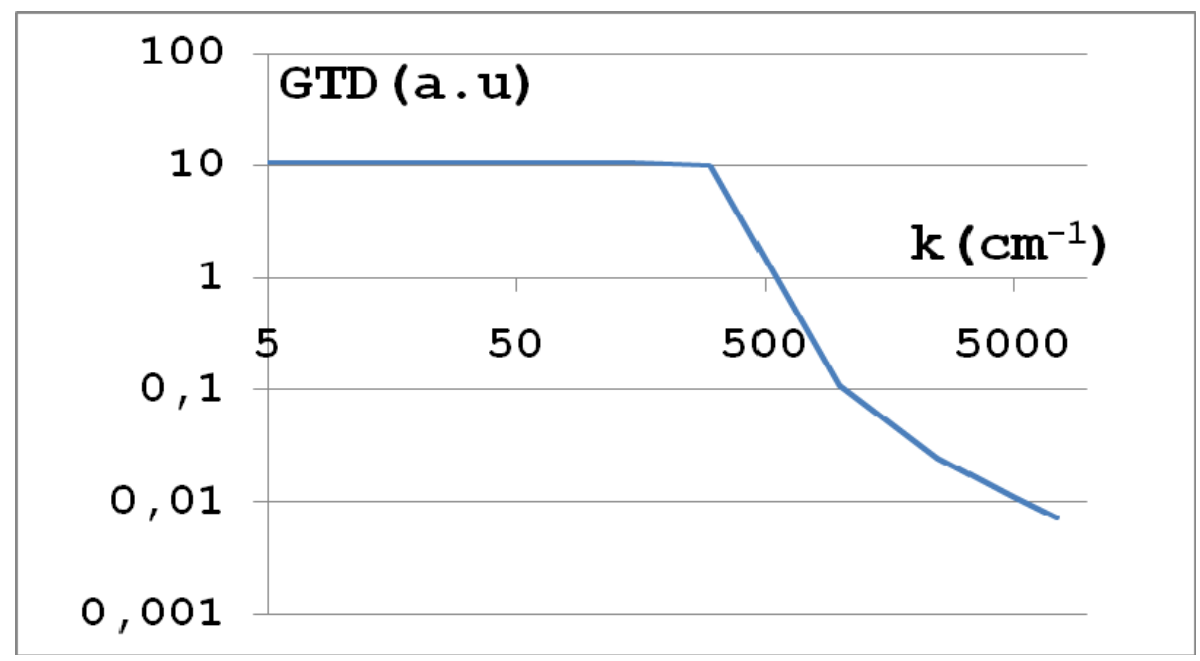

Figure 4. GTD as a function of coupling coefficient $(k)$ for a LCG of length $15 \mathrm{~cm}$ and chirp $C=100 \mathrm{~nm} / \mathrm{cm}$. The values of GTD are normalized so that each unit corresponds to a phase derivative $\mathrm{d} \varphi_{\mathrm{BG}} / \mathrm{d} \lambda$ of $1 \mathrm{rad} / \mathrm{nm}$. An asymptotical approach to zero is observed for big values of $k$. 


\section{ALGORITHMS}

\subsection{Genetic Algorithm}

The genetic algorithm ${ }^{2}$ is a fast way of approaching solutions when the space of variables is so large that an exhaustive search becomes impossible. In our case the variables are represented by the physical characteristics of the grating's layers, each of the layers having its own thickness value and/or its own value of refractive index. The genetic algorithm finds very rapidly an optimal solution for these variables according to a fitness function which it uses for evaluation. Its disadvantage is that it does not find a general optimal solution, but only local maxima of the fitness function, depending on the random initial population used by the algorithm. The precise algorithm used for the following simulations starts by selecting an initial gratings population of size $N$, with each of the individual gratings having $M$ layers with random refractive indices $n(i, j)$ and/or random thicknesses $l(i, j)$, where $i=1,2, \ldots, N$ and $j=1,2, \ldots, M$. Each individual grating is given a value according to the fitness function $F$ chosen to suite the purpose of the algorithm. In this particular case, the fitness function should take into account the amplitude and phase of the reflectivity of each grating. The gratings which have high values of reflectivity and in the same time a negative value of the group time delay (negative phase derivative) in the desired bandwidth, those gratings will have high values of the fitness function. The probability of using each grating as a parent in the next generation is proportional to the fitness function. A wheel of fortune algorithm is used to extract $N$ parent-gratings. On this virtual wheel of fortune, each grating occupies a slice proportional to its fitness function. Thus, it is obvious that the gratings with higher values of the fitness function will be extracted more than once by the algorithm. The parents will then be coupled two by two, and each pair of parent-gratings will give rise to a new pair of child-gratings. There are more methods of combining the layer-characteristics of the parent-gratings to obtain the children, but only one was used in this paper, namely the uniform crossover. This method consists in choosing for each child layer the corresponding layer of one of its parents, where the binary choice of the parent is done randomly. As an example, let us consider the case of two parents having the layers' lengths $\left\{l\left(\mathrm{i}_{1}, 1\right) ; l\left(\mathrm{i}_{1}, 2\right) ; l\left(\mathrm{i}_{1}, 3\right) ; l\left(\mathrm{i}_{1}, 4\right) ; \ldots ; l\left(\mathrm{i}_{1}, M-1\right)\right.$; $\left.l\left(\mathrm{i}_{1}, M\right)\right\}$ and $\left\{l\left(\mathrm{i}_{2}, 1\right) ; l\left(\mathrm{i}_{2}, 2\right) ; l\left(\mathrm{i}_{2}, 3\right) ; l\left(\mathrm{i}_{2}, 4\right) ; \ldots ; l\left(\mathrm{i}_{2}, M-1\right) ; l\left(\mathrm{i}_{2}, M\right)\right\}$. A possible combination of these two parents, using the uniform crossover method, may be $\left\{l\left(\mathrm{i}_{1}, 1\right) ; l\left(\mathrm{i}_{2}, 2\right) ; l\left(\mathrm{i}_{2}, 3\right) ; l\left(\mathrm{i}_{1}, 4\right) ; \ldots ; l\left(\mathrm{i}_{2}, M-1\right) ; l\left(\mathrm{i}_{2}, M\right)\right\}$ and $\left\{l\left(\mathrm{i}_{2}, 1\right) ; l\left(\mathrm{i}_{2}, 2\right) ; l\left(\mathrm{i}_{1}, 3\right)\right.$; $\left.l\left(\mathrm{i}_{2}, 4\right) ; \ldots ; l\left(\mathrm{i}_{1}, M-1\right) ; l\left(\mathrm{i}_{2}, M\right)\right\}$. Very rarely, a mutation could be introduced at a random position in the characteristics of the child-gratings. Having obtained a new population of $\mathrm{N}$ child-gratings, the algorithm can repeat itself until a fitness function $F$ greater than a desired value is obtained or until a maximum number of iterations has been reached.

\subsection{Discrete Layer Peeling}

The DLP algorithm ${ }^{3}$ is a method first adapted by Feced et al. ${ }^{4}$ from Digital Signal Processing (DSP) techniques of filter design $^{5}$. It uses an Inverse Fourier Transform to translate into time domain an ideal frequency response. Once in time domain, the response is passed through different functions (truncating, windowing and shifting) in order to become realizable. The time response determines ${ }^{3}$ the physical structure of the grating, so that is why in this stage the feasibility of a grating is decided. This correlation between the time domain and the physical structure is possible because as the signal propagates through the grating the time response (in this case the reflected signal) changes accordingly. The realizable time response is then translated back into frequency domain by a Fourier Transform. Thus by starting with a desired frequency response it can be arrived, after a series of mathematical manipulations, to a realizable frequency response. Not every response is possible, and the mathematical reason for this is that certain time responses are anticausal and thus could not be obtained by the use of real physical structures. The method of Feced et al. ${ }^{4}$ for making a frequency response realizable equates thus with transforming its time response from an anti-causal to a causal one. Sadly, it can not be applied directly for this purpose, as by shifting the time response the phase derivative (thus the GTD) changes also. Feced's method is suitable when the phase of the frequency response is not important and the amplitude is the only one that counts. That is why the approach of this paper was different. It is based on a mathematical treatment known as zero-padding method ${ }^{5}$ which is used by engineers in DSP for many years. Its purpose is to detect if the GTD of the real structure created by the DLP algorithm is the same as the desired one. A short explanation of how precisely it does that follows next. To specify a desired analogue frequency response in the computer's language, a process of sampling and quantization must first be employed. Let $Q$ be the number of points used for the sampling of the desired frequency response. When using an Inverse Discrete Fourier Transform (IDFT) the $Q$ complex numbers will correspond to approximately $2 Q$ real numbers representing the time domain. By zero-padding this signal at the right ${ }^{5}$ with a number $2 Q$ of zeros and then translating the entire $4 Q$ signal back to frequency domain, what it will be obtained is a quantity of $2 Q$ complex numbers representing the same frequency response as the initial one, only now having twice the initial resolution. If the initial frequency response corresponds to a realizable (causal) signal, the process of zero-padding at the right will not change the form of the signal, no matter how much the resolution is increased. 


\section{SIMULATION RESULTS}

\subsection{Genetic Algorithm}

As it was explained in section 3.1, choosing the right fitness function $F$ is vital for the outcome of the genetic algorithm. Additionally, the complexity of $F$ dictates the speed of the algorithm, thus a good fitness function must be as simple as possible to be efficient, as it is calculated $N$ times for each iteration. Although many fitness functions were tested, a representative example is shown in Fig. 5, for which

$$
F=\sum R|\mathrm{~d} \varphi / \mathrm{d} \lambda|, \quad \text { if } \mathrm{d} \varphi / \mathrm{d} \lambda<0,
$$

where $R$ is the reflectivity. The summing depends on the number of sampling points used by the algorithm and it takes place only for wavelengths for which the GTD is negative $(\mathrm{d} \varphi / \mathrm{d} \lambda<0)$. Despite the fact that $F$ should promote simultaneous encounters of a negative GTD and a high reflectivity, it can be seen in Fig. 5 that the regions of negative GTD (bottom arrows) coincide only with minima of reflectivity (top arrows). There can be used numerous other fitness functions, but none of them showed better results than the simple example of (3). To analyze the reasons for not having an occurrence of both a negative GTD and a maximum of reflectivity, the Discrete Layer Peeling (DLP) method will be tried in the next section. The physical structure which corresponds to Fig. 5a) is shown in the top of Fig. 6. As it can be noticed, the layers of the resulting grating have random thicknesses, but a constant refractive index variation. The same can be said for the random Bragg grating shown in the middle of Fig. 6 which has the spectrum of Fig. 5b). For better clarity, a zoom on this second structure has been made in the interval going from $50 \mu \mathrm{m}$ to $80 \mu \mathrm{m}$ and represented at the bottom of Fig. 6. It is worth stating here that simulations in which both the layers' lengths and refractive indices were let to vary randomly were not qualitatively superior to simulations in which only the layers' lengths were set to vary randomly (as the case of Fig. 5 and 6).

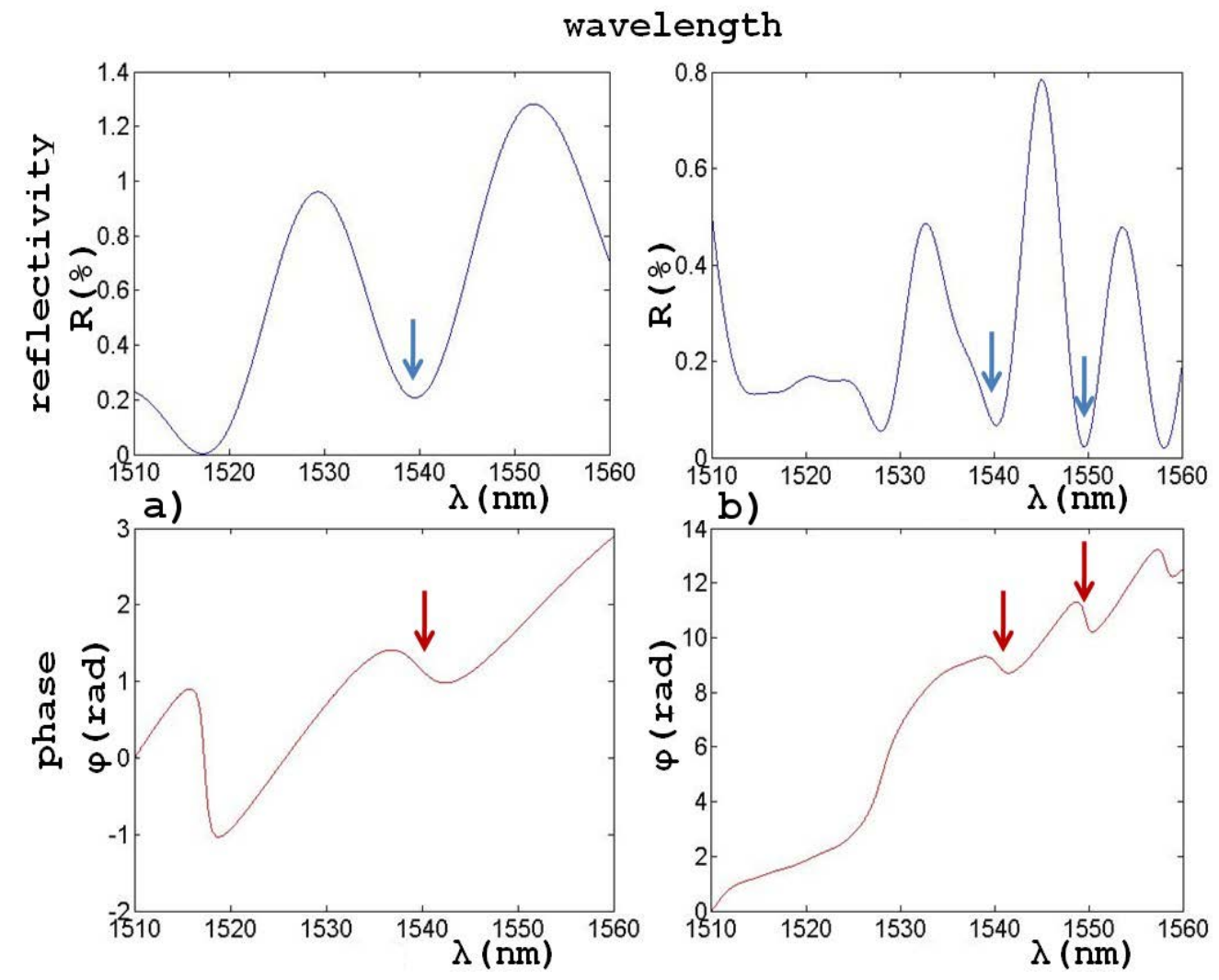

Figure 5. Reflectivity (top) and phase (bottom) are plotted as a function of $\lambda$ for the case of two gratings obtained by

Genetic Algorithm. The number of layers for each Bragg grating is: a) 100 and b) 300 . 

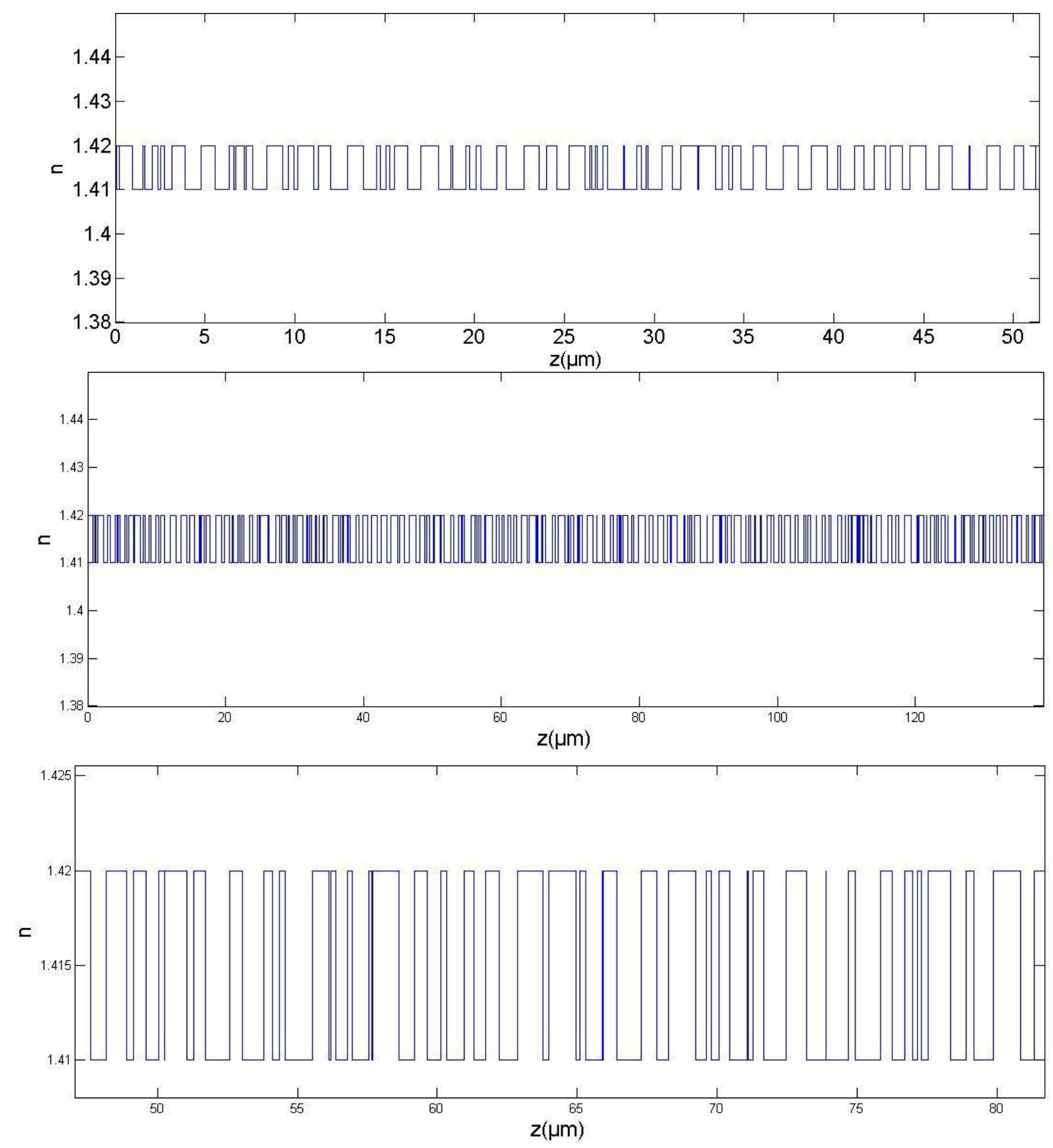

Figure 6. Refractive index profiles of the structures corresponding to the reflectivity spectra shown in Fig. 5a (top) and Fig. 5b (middle). The bottom picture consists of a zoom onto the middle structure, showing more clearly the random character of the layers' thicknesses.

\subsection{Discrete Layer Peeling}

As explained in section 3.2, this method starts with a desired frequency response. In this particular case, the desired frequency response consists of having a maximum of amplitude simultaneously with a negative phase derivative. As explained in 3.2, the frequency domain signal is entered as a set of $Q$ complex numbers, having both amplitude and phase, which were sampled from the desired analogue response. After transforming this frequency signal in the time 
domain, a set of $2 Q$ real numbers is obtained, which contains enough information for the construction of a grating structure by $\mathrm{DLP}^{3}$. At this point though, there is the possibility that the reflectivity spectrum returned by this structure coincides with the desired spectrum only in the sampling points, and that between these points its behavior can be completely erratic. To check if this is the case, the zero padding method is used, increasing the resolution (as explained in section 3.2) until a stable figure is obtained. As it can be seen in Fig. 7, only desired gratings having a positive GTD (dashed line: $\mathrm{GTD}_{\text {des }}>0$ ) are possible (on the dashed line $\mathrm{GTD}_{\text {real }}=\mathrm{GTD}_{\text {des }}$ ). Meanwhile, each time the DLP algorithm is applied to design a high reflectivity Bragg grating with a negative GTD (solid line: $G_{T D} D_{\text {des }}<0$ ) in the reflection bandwidth, we are in fact designing a grating with a positive GTD (on the solid line $\mathrm{GTD}_{\text {real }}>0$ ).

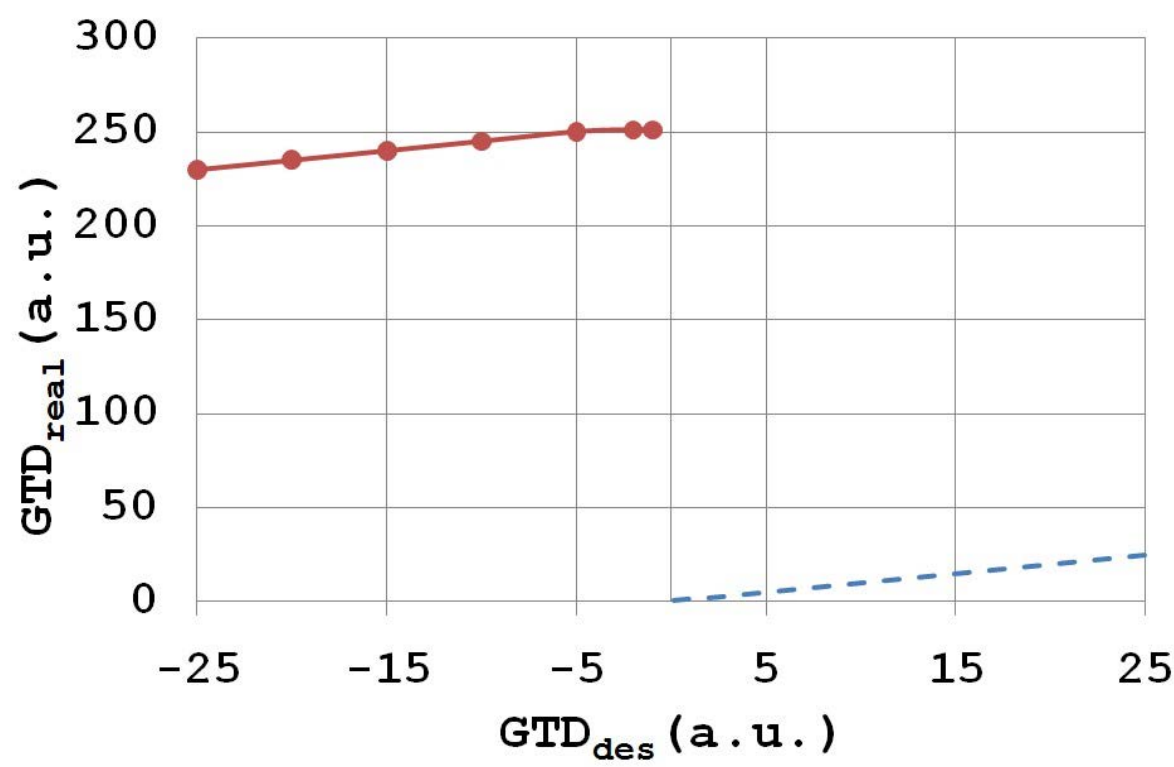

Figure 7. Real (anti-aliased) normalized GTD (GTD ${ }_{\text {real }}$ ) as a function of desired normalized GTD (GTD des) for a grating synthesized by the DLP method. Reflectivity is considered to be maximal for every simulation point. The values of GTD are normalized so that each unit corresponds to a phase derivative $d \varphi_{\mathrm{BG}} / \mathrm{d} \lambda$ of $1 \mathrm{rad} / \mathrm{nm}$.

\section{CONCLUSION}

From our knowledge, we tried for the first time to extend the bandwidth of resonant modes in a Fabry-Perot cavity by Bragg grating engineering (Fig. 1). We demonstrated that for this to happen, the designed grating must have a negative GTD. Sadly, this also implies (Fig. 7) the impossibility to simultaneously have a maximum of reflectivity. We have nevertheless obtained a few practical examples of negative GTD gratings (having though small values of reflectivity) by way of Genetic Algorithm, of which two examples are shown in Fig. 6 (for which the reflectivities are shown in Fig. 5).

\section{ACKNOWLEDGEMENT}

The authors would like to thank the "Conseil Regional de Lorraine” (France) for financial support of this project. This work is also financially supported by the French National Research Agency (ANR) grant through the CONTINUUM project ANR-09-NANO-035 01. All partners of this project are kindly acknowledged. 


\section{REFERENCES}

[1] Bergonzo, A., Gohin, E., Landreau, J., Durand, O., Brenot, R., Duan, G-H., and Jacquet, J., “Tuning Range Extension by Active Mode-Locking of External Cavity Laser Including a Linearly Chirped Fiber Bragg Grating”, IEEE Journal of Selected Topic in Quantum Electronics, Vol. 9, n5, P 1118, September/October (2003).

[2] Skaar, J., Risvik, K. M., "A Genetic Algorithm for the Inverse Problem in Synthesis of Fiber Gratings," Journal of Lightwave Technology, vol. 16, no. 10, October 1998.

[3] Skaar, J., Wang, L., Erdogan, T., “On the Synthesis of Fiber Bragg Gratings by Layer Peeling,” IEEE Journal of Quantum Electronics, February 2001.

[4] R. Feced, M. N. Zervas and M.A. Muriel, "An efficient inverse scattering algorithm for the design of nonuniform fibre Bragg gratings” J. Quantum El., vol. 35, 1999, pp. 1105-1115.

[5] Smith, S. W., [The Scientist and Engineer Guide to Digital Signal Processing], California Technical Publishing, 1997-2011.

[6] Buus, J., [Single Frequency Semiconductor Lasers], SPIE Press, 25-27 (1991)

[7] Katsidis, C.C., and Siapkas, D.I., 'General Transfer-Matrix Method for Optical Multilayer Systems with Coherent, Partially Coherent, and Incoherent Interference', Applied Optics, Vol. 41, Issue 19, pp. 39783987 (2002) 